\title{
OVERVIEW OF CURRENT DENSITY MEASUREMENTS AND SAWTOOTH STUDIES ON TEXTOR
}

\author{
H. R. KOSLOWSKI* Institut für Plasmaphysik, Forschungszentrum Jülich GmbH \\ EURATOM Association, Trilateral Euregio Cluster, D-52425 Jülich, Germany
}

Received April 29, 2004

Accepted for Publication July 18, 2004

The analysis of Faraday rotation measurements shows that the safety factor on the magnetic axis stays well below one during the full sawtooth cycle.

The current density profile has a unique shape during the sawtoothing plateau phase of ohmic discharges, independent of plasma current and magnetic field, when it is plotted in suitable normalized coordinates.

The observation of short lasting spikes on the Faraday rotation signals can be interpreted as the formation of a transient current sheet during the sawtooth crash.

Direct measurements of the magnetic amplitude of the internal kink mode with a fast polarimeter channel show that the displacement of the core because of the growing precursor mode stays much smaller than the $q=1$ radius just prior to the sawtooth collapse.

The versatile neutral beam heating system on Tokamak EXperiment for Technology Oriented Research (TEXTOR) allows the study of the influence of the plasma rotation on the sawtooth period, which has a minimum when the magnetohydrodynamic rotation in the tokamak frame is stopped.

KEYWORDS: FIR polarimetry, current density, sawteeth

\section{INTRODUCTION}

The combined HCN-interferometer/polarimeter diagnostic on the Tokamak EXperiment for Technology Oriented Research (TEXTOR) tokamak was operational from the start of the experimental program. TEXTOR was the first fusion experiment with a routinely operated current profile diagnostic based on the Faraday rotation effect. A short description of the system and an overview on the available diagnostics on TEXTOR is given in this issue. ${ }^{1}$ A more detailed description of the instrument can

\footnotetext{
*E-mail: h.r.koslowski@fz-juelich.de
}

be found elsewhere. ${ }^{2}$ The TEXTOR setup, designed following a proof-of-principle test of the Faraday rotation measurement on Tokamak in Fontenay-aux-Roses ${ }^{3}$ (TFR), features nine channels in one poloidal cross section of the torus, which allows the reconstruction of local quantities from the line-integrated signals of electron density and parallel poloidal field component.

Besides the determination of the current density distribution itself, which could be inferred before only from the temperature profile using the assumption of neoclassical conductivity, the analysis of specific features of the current density profile during transient events like sawteeth was of special interest.

The sawtooth instability of tokamak plasmas ${ }^{4}$ had been found in 1974, only a few years before the current density measurement became operational, and a direct test of the common theoretical explanation ${ }^{5}$ was still missing. The TEXTOR result, that the change in the central safety factor was smaller than widely expected and $q_{0}$ stays below one during the full sawtooth cycle, was later confirmed by polarimetry measurements on the Joint European Torus ${ }^{6}$ (JET) and verified by simultaneous motional Stark effect (MSE) measurements on JET (Ref. 7). Further corroboration came from far infrared (FIR) polarimetry measurements on the Microwave Tokamak eXperiment $^{8,9}$ (MTX), MSE measurements on the Tokamak Fusion Test Reactor ${ }^{10}$ (TFTR), and polarimeter measurements on TEXT-Upgrade. ${ }^{11}$ The first MSE measurements on DIII-D yielded similar results, ${ }^{12}$ but a later paper stated that $q_{0}$ after the sawtooth collapse increased to one..$^{13}$ As a consequence, the model of full reconnection of the magnetic flux within the $q=1$ surface during the fast sawtooth crash as well as the quasi-interchange model, ${ }^{14}$ which requires $q_{0}$ close to one and only a very small change during the sawtooth crash, were questioned by these results.

Current density measurements were performed for a variety of discharge conditions, giving important results for the interpretation of specific features observed, e.g., the development of negative central shear after impurity accumulation ${ }^{15}$ or the peaking of the current profile during high confinement, radiative improved (RI)-mode discharges. ${ }^{16,17}$ 
Additional experimental investigations of the sawtooth phenomenon were performed. The addition of a fast polarimeter channel enabled the measurement of the magnetic amplitude of the internal kink mode. The results corroborated the previous finding of incomplete reconnection during the sawtooth collapse. Moreover, the detailed analysis of perturbations of the Faraday rotation signal during the sawtooth crash revealed certain structures that point to the formation of a transient current sheet during the sawtooth crash. Both findings may be important for the understanding of the sawtooth mechanism itself. ${ }^{18}$

In connection with the versatile neutral beam injection system on TEXTOR, which allows precise control of the injected power and therefore the momentum exerted on the plasma, the dependence of the sawtooth period on plasma rotation was determined. This subject has recently gained new attention because sawteeth are regarded as the most important trigger of neoclassical tearing modes in ITER.

In the following sections the aforementioned topics are outlined, and the most important findings are recalled together with a short description of the specific experiments performed.

\section{EXPERIMENTAL RESULTS}

In a tokamak plasma the distribution of the plasma current plays an important role because the resulting poloidal magnetic field determines the confinement properties and is crucial for the stability of the tokamak plasma. The appearance and growth of numerous instabilities are closely connected to the existence of certain rational surfaces in the plasma as well as subtle local modifications of the poloidal magnetic field. Especially the sawtooth instability ${ }^{4}$ which manifests itself in periodic collapse events of the plasma density and temperature in the center, depends on the existence of the rational $q=1$ surface in the plasma. It is, therefore, not surprising that the availability of a routine plasma current diagnostic promoted work in this specific area.

\section{II.A. Unique Shape of the Current Density Distribution}

The first measurements with the polarimeter on TEXTOR were done in ohmic plasmas because auxiliary heating systems were not available in the beginning. These measurements were carried out with special attention to the possible errors imposed by the required inversion of the line-integrated measurements of electron density:

$$
n_{e, \text { line }}(R)=\int n_{e}(R, z) d z
$$

and Faraday rotation angle

$$
\alpha=c_{F} \int n_{e}(R, z) \mathbf{B}_{\mathbf{p}}(R, z) \cdot \mathbf{d z},
$$

with

$$
c_{F}=\left(e^{3} \lambda^{2}\right) /\left(8 \pi^{2} \epsilon_{0} m_{e}^{2} c^{3}\right),
$$

where

$$
\begin{aligned}
\lambda & =\text { wavelength of the laser } \\
e & =\text { electron charge } \\
m_{e} & =\text { electron mass } \\
c & =\text { speed of light } \\
\epsilon_{0} & =\text { permittivity of free space. }
\end{aligned}
$$

The integration along $z$ direction is because of the vertical probing beam arrangement on TEXTOR. The poloidal magnetic field profile, which is the quantity derived from the latter measurement, therefore relies on two inversions because the local electron densities, determined from interferometry, are required for data evaluation.

Ohmic TEXTOR discharges are highly reproducible, i.e., for carefully chosen operating conditions the time traces of measured signals showed no significant differences between pulses. The circular plasma cross section, determined by a set of poloidal limiters, allowed small shifts of the horizontal plasma position in a series of discharges. By shifting the plasma horizontally by $2 \mathrm{~cm}$ from discharge to discharge, the number of probing channels could be multiplied. Using five plasma positions, equivalent to a plasma shift of $\pm 4 \mathrm{~cm}$ and keeping the plasma diameter constant by adjustment of the limiter configuration, the number of effective channels could be enhanced up to 45. All measured data fitted without noticeable deviations on a unique curve, which proved that the spline interpolation applied in situations in which these detailed scans could not be performed, were valid. Based on this data, the $q$ profile with an uncertainty of $\sim 15 \%$ for the central value and 5 to $10 \%$ at half radius was derived. ${ }^{19}$

The analysis for ohmic discharges (at different plasma currents and magnetic fields) revealed that the current profile during the sawtoothing flattop phase can be prescribed by a unique formula, using a normalization for the radial coordinate and the current density. ${ }^{18,20}$ Figure 1 shows the experimental profiles (left) plotted in normalized radius $r^{*}=r /\left(\mu_{0} R I / B_{t}\right)^{1 / 2}$ and normalized current density $j^{*}=j /\left(B_{t} / \mu_{0} R\right)$. The right part of the figure is a comparison of the fitted curve with the ohmic current profiles from other tokamaks (derived from the electron temperature profiles assuming neoclassical conductivity ${ }^{21}$ ), which show good agreement with the normalized current profile shape derived on TEXTOR. It is important to note that the above result holds for the flattop phase of ohmic discharges when regular sawtooth activity limits the central current density. The application of auxiliary heating and noninductive current drive allows the decoupling of heating and current profiles and 

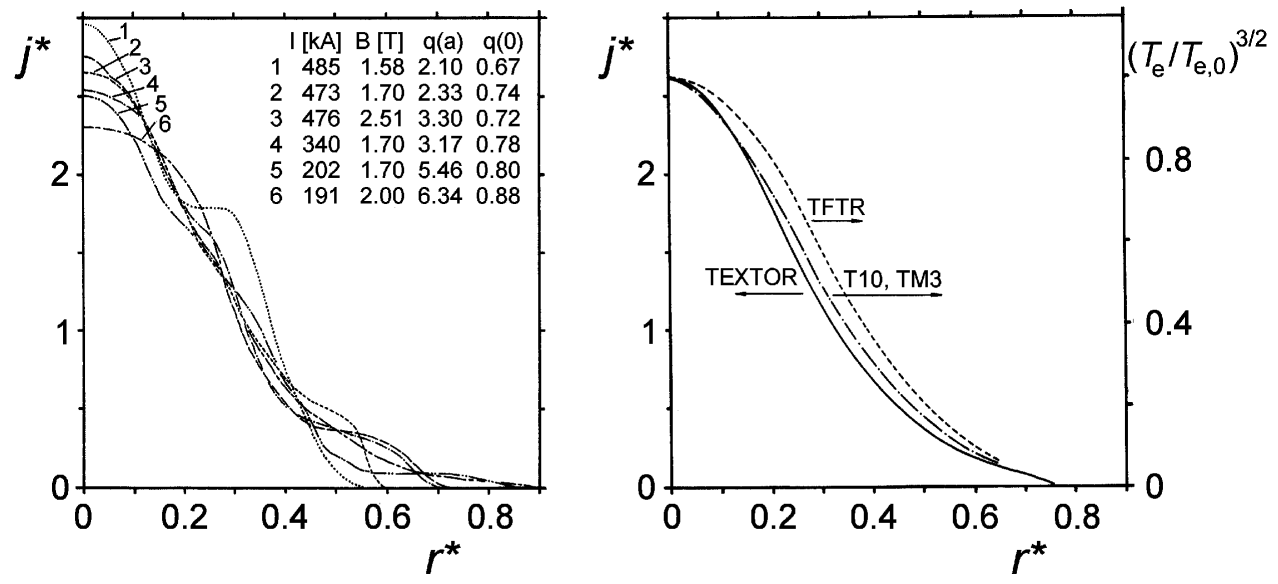

Fig. 1. Normalized current density profiles for TEXTOR (Ref. 18). The left figure shows the experimental profiles for various plasma parameters. The right part displays the fitted universal profile (solid line) and compares it to current density profiles from other tokamaks.

leads to a variety of current profiles that cannot anymore be prescribed by such a unique shape.

\section{II.B. Evolution of the Central Safety Factor During the Sawtooth Crash}

For the analysis of the change in $q_{0}$ because of the sawtooth crash, the high reproducibility of TEXTOR discharges has been utilized in a second way. The time traces of the interferometer and polarimeter measurements are chopped into small parts, each one representing exactly one sawtooth period. All these individual sawtooth periods are interpolated on the same (relative) time axis and summed up afterward. As a result, smoothed data representing one sawtooth cycle during the plateau phase of an ohmic discharge is obtained. This procedure effectively reduces the noise on the Faraday rotation signals without reducing the time resolution of $\sim 1 \mathrm{~ms}$. After inversion of the data, the temporal evolution of the current density distribution was obtained. The average value of the safety factor on axis $q_{0}$ for a standard ohmically heated $(\mathrm{OH})$ discharge $\left(B_{t}=2 \mathrm{~T}, I_{p}=350 \mathrm{kA}, \overline{n_{e}}=3 \times 10^{19} \mathrm{~m}^{-3}, T_{e}=\right.$ $1 \mathrm{keV}$, and circular shape) was determined to be 0.77 $( \pm 0.12)$, and the relative change during the sawtooth crash amounts to $8 \%$ (Ref. 22), i.e., $q_{0}$ stays well below one during the whole sawtooth period.

In a low beta plasma like the $\mathrm{OH}$ discharges under investigation, the position of the magnetic axis can be readily determined from the zero crossing of the Faraday rotation profile. A periodic movement of the equilibrium position of the magnetic axis with an amplitude of $1 \mathrm{~mm}$ was determined. During the slow ramp phase of the sawtooth, the magnetic axis moves outward, i.e., to the lowfield side of the torus, because of the increasing plasma pressure when the density and temperature profiles peak inside the $q=1$ surface. The initial position is restored during the sawtooth crash as a result of the pressure loss.

\section{II.C. Formation of a Current Sheet During the Sawtooth Crash}

The analysis of the sawtooth-averaged data set, in which the number of probing beams has been virtually increased and the noise level was substantially decreased by coherent averaging in phase with the sawtooth period, shows another very interesting feature. ${ }^{18}$ The probing beams located near the outboard side of the $q=1$ surface, i.e., close to $R=R_{0}+r_{q=1}$, exhibit small spikes during the sawtooth crash. Because the interferometric data, which have been processed in the same way, do not show this feature, it has been attributed to a transient perturbation of the poloidal magnetic field during the sawtooth crash. Two adjacent probing beams, which intersect the plasma close to the $q=1$ surface on the low-field side, show this perturbation with opposite signs. There were indications for a much weaker effect on the high-field side of the torus, too. This can be explained by a current sheet in between both probing beams having an $m=1, n=0$ spatial structure. The absence of a helical twist of the induced current sheet was concluded from the fact that the coherent averaging of more than 100 individual sawtooth periods resulted in an almost complete suppression of the precursor oscillations on the interferometer channels, i.e., the perturbations on the polarimeter measurements are not related to the phase and helical structure of the $m=1, n=1$ sawtooth precursor. ${ }^{23}$ The required current to explain the measured amplitude of the spike on the polarimeter signals is on the order of $3 \mathrm{kA}$ (Ref. 23), which is $\sim 1 \%$ of the total plasma current of $380 \mathrm{kA}$.

A theoretical model in terms of a Pfirsch-Schlüter current has been given. ${ }^{24}$ This current is caused by a transient steepening of the pressure gradient when the transport within the $q=1$ surface is enhanced during the fast sawtooth collapse, flattening the pressure inside the rational surface, and increasing the gradient outside. 
Irrespective of the underlying mechanism, the observed $m=1, n=0$ magnetic field perturbation together with the $m=1, n=1$ structure of the sawtooth (precursor) mode will cause stochastization ${ }^{25}$ of the magnetic field and thus contribute to the rapid expulsion of heat and particles from the plasma center.

\section{II.D. Determination of Characteristic Features of the $q$-Profile for Various Discharge Conditions}

Following the first measurements of the safety factor in sawtoothing $\mathrm{OH}$ discharges, the polarimeter has been applied routinely to a wide variety of discharge conditions. An example of the high reliability of the measured data, even in situations that cannot be easily repeated to allow data averaging or the addition of virtual probing beams, is depicted in Fig. 2 (Ref. 16). The figure shows three $q$ profiles measured at different times within one discharge. The plasma was heated by counter neutral beam injection, and a frequent observation under those discharge conditions was the stabilization of sawtooth oscillations. The reason for this is still unclear because this behavior was not found to be general but occurring from time to time and has been attributed to the accumulation of light impurities in the plasma center. In discharge 65719 , sawtooth oscillations were still present at $t=1.6 \mathrm{~s}$. The safety factor profile (averaged over $100 \mathrm{~ms}$, which is larger than an individual sawtooth period) is below one on the axis, and a $q=1$ surface is established in the plasma. At $t=2.0 \mathrm{~s}$, the last sawtooth occurs. The measurement of the line-averaged electron density at $R=1.75 \mathrm{~m}$, i.e., close to the magnetic axis, exhibits clearly visible precursor oscillations, which means that

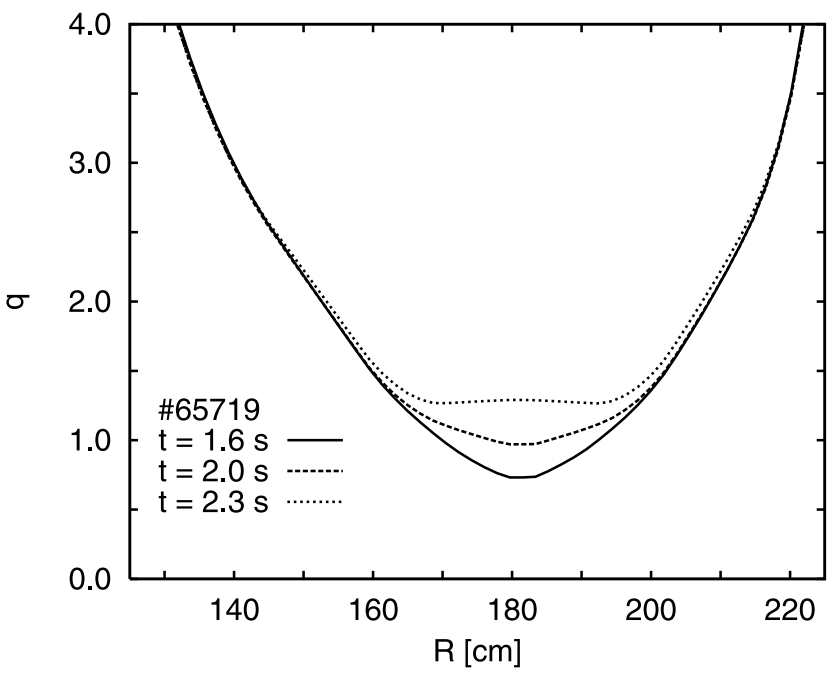

Fig. 2. Measured safety factor profiles in a discharge where sawteeth disappear. ${ }^{16}$ The profiles are during the sawtoothing phase $(1.6 \mathrm{~s})$, when the last sawtooth is observed $(2.0 \mathrm{~s})$, and without sawteeth $(2.3 \mathrm{~s})$. the probing chord passes through a region close to the $q=1$ radius. This is in good agreement with the result from the polarimeter, which shows that $q$ on the axis is close to one and the radius of the $q=1$ surface is quite small. The last profile shown at $t=2.3 \mathrm{~s}$ is measured during the phase when sawteeth were suppressed. Again, the reconstructed profile shows good agreement with the experimental observation. The $q$ profile is well above unity, consistent with sawtooth suppression.

The measurements of the current density in TEXTOR were useful also for the understanding of transport. The high confinement regime in TEXTOR is the RI mode. ${ }^{26}$ A quite extensive modeling has been done for these plasmas. One of the results was that because of the cooling of the plasma edge by injection of light impurities (neon, silicon), a steepening of the current density profile was anticipated. ${ }^{17}$ Measurements of the current profile in RI-mode plasmas did confirm the code calculations. ${ }^{16}$

Another interesting result was obtained during experiments with high-Z limiters on TEXTOR ${ }^{15}$ In these plasmas the accumulation of sputtered atoms from the limiters was observed for unfavorable discharge conditions. As a consequence, a strong plasma instability, resembling a sawtooth with a larger inversion radius, did occur. Measurements with the polarimeter revealed that because of the influx of the strongly radiating impurities, which cooled down the plasma center, the current on the axis decreased and a $q$ profile with negative shear developed. The observed crash-like relaxation events were attributed to the coupling and spatial overlap of double tearing modes of different helicity $(2 / 1$ and $3 / 2)$, giving a short circuit for the inner part of the plasma. The measured current density profile corroborated this hypothesis, and modeling with the MHD code ${ }^{27}$ CASTOR, using the experimental profiles of safety factor and plasma pressure, showed that indeed both double tearing modes were unstable. $^{28}$

\section{II.E. Measurement of the Magnetic Amplitude of the Sawtooth Precursor Mode}

Aimed on a direct measurement of the magnetic amplitude of the sawtooth precursor mode (the internal kink mode), the polarimeter has been extended by a horizontally measuring channel. The laser beam enters the plasma on the low-field side in the equatorial plane, traverses the plasma, and is reflected under a small toroidal angle by a mirror on the high-field side. The arrangement ensures that the component of the toroidal field in the direction of the incoming and outgoing probing beams is canceled; and because of the up-down symmetry, the unperturbed poloidal field is always perpendicular to the beam. Any measured signal is therefore because of magnetic field perturbations; especially the internal kink mode can be detected. The time resolution of this probing beam was increased up to $10 \mu$ s by the application of a fast detector. 
The new polarimeter channel was able to detect the magnetic perturbation of the sawtooth precursor. Figure 3 shows the measured Faraday rotation angles (the values of the maxima of the precursor oscillation are measured) plotted versus the time difference to the following sawtooth crash. ${ }^{29}$ An exponential growth of the $m=1$ kink amplitude could be determined in an ohmic standard discharge until very shortly before the collapse. The maximum amplitude of $0.26 \mathrm{deg}$ corresponds to an average perturbation field of $\overline{B_{1}}=13 \mathrm{mT}$. From this value the displacement of the magnetic axis was determined to be $\sim 5 \mathrm{~cm}$, which amounts to approximately one-third of the $q=1$ radius. ${ }^{23}$ This result is consistent with the earlier finding of incomplete reconnection during the sawtooth crash.

\section{II.F. Dependence of the Sawtooth Period on Plasma Rotation}

As a last example for sawtooth studies carried out on TEXTOR, the dependence of the sawtooth period on the toroidal plasma rotation is shown in Fig. 4. TEXTOR has two neutral beam injectors, one injecting the particles in the direction of the plasma current (co) and the other in the opposite direction (counter). The neutral beam power is up to $1.5 \mathrm{MW}$ for each injector and can be controlled very precisely either by changing the acceleration voltage or by partly closing an aperture in the beam line. The second approach allows the control of the power down to zero without changing the energy of the beam particles, i.e., the penetration depth stays constant. By changing the beam power, the momentum exerted on the plasma

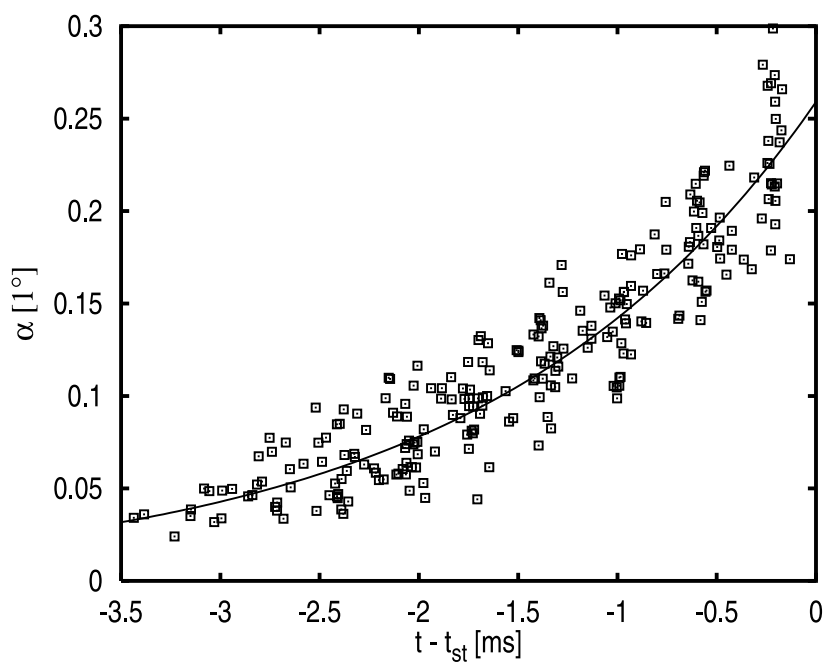

Fig. 3. Amplitude of the Faraday rotation angle (proportional to the magnetic amplitude of the $m=1$ kink) plotted versus the time prior to the next sawtooth crash. ${ }^{29}$

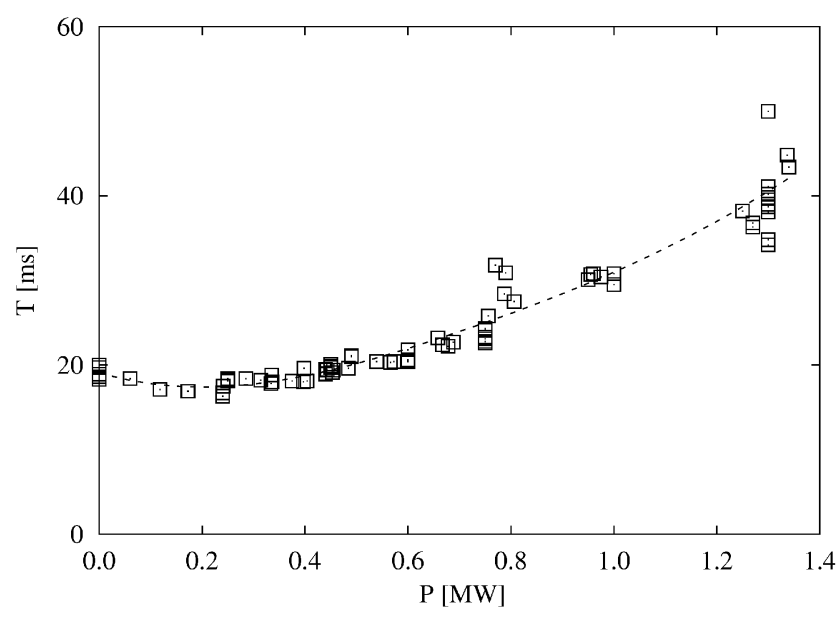

Fig. 4. Sawtooth period versus the heating power of the neutral beam coinjector.

column is varied, and as a result the toroidal rotation velocity is changed.

It was found experimentally that the direction of the rotation of MHD modes in the plasma center, e.g., the sawtooth precursor mode, rotates in a pure ohmic plasma opposite the plasma current (note that the MHD frequency is the sum of the plasma rotation frequency and a diamagnetic contribution). Therefore, a small amount of momentum input in the direction of the plasma current is required to stop the MHD rotation. This could be monitored, e.g., by the frequency of the sawtooth precursor, which goes to zero. The required power of the coinjecting neutral beam is 200 to $250 \mathrm{~kW}$.

Figure 4 shows the sawtooth period plotted versus the power of neutral beam coinjection that has been varied using the beam aperture method. It can be seen that the sawtooth period has a slight minimum at the same power required to stop the MHD rotation of the plasma. This indicates that the rotation in the core has a stabilizing influence on sawteeth and that nonrotating plasmas are more susceptible to the sawtooth instability. Because the total heating power was varied during this scan as well, there could be some influence on sawtooth stability resulting from the increase of plasma beta. In addition, a qualitatively similar result has been obtained in plasmas in which the sum of the injected power from both beams was kept constant and only the ratio between co- and counter-injection was varied. A similar experiment was recently carried out at the JET tokamak, which gave qualitatively the same result, i.e., the sawtooth period shows a minimum at a definite toroidal rotation speed. ${ }^{30}$ This result is important for a next-step fusion device because sawteeth with long periods are known to be an effective trigger of neoclassical tearing modes; and means to avoid triggering this mode, for example, keeping the toroidal rotation low, are required. 


\section{SUMMARY AND CONCLUSION}

The aforementioned examples have shown that interesting and important results were achieved from current density measurements on TEXTOR. The simple circular plasma shape and the large number of reliable core diagnostics were beneficial in gaining the data and doing the analysis.

Faraday rotation measurements showed that the safety factor on the magnetic axis stays well below one during the full sawtooth cycle, i.e., full reconnection during the sawtooth crash is very unlikely to occur.

The current density profile is well fitted by a unique shape during the sawtoothing plateau phase of ohmic discharges, independent of plasma current and magnetic field, when plotted in suitable normalized coordinates.

The observation of short lasting spikes on the Faraday rotation signals was interpreted as the formation of a transient current sheet during the sawtooth crash driven by a Pfirsch-Schlüter current because of a transient steepening of the pressure profile.

A fast polarimeter channel (time resolution up to 10 $\mathrm{kHz}$ ) allowed the direct measurement of the magnetic amplitude of the sawtooth precursor mode and deduced the shift of the magnetic axis. The displacement of the core because of the growing precursor mode stays much smaller than the $q=1$ radius until very close to the sawtooth collapse.

The versatile neutral beam heating system on TEXTOR permitted the study of the influence of the plasma rotation on MHD stability, especially the internal kink mode. The sawtooth period has a minimum when the MHD rotation in the tokamak frame is stopped.

\section{ACKNOWLEDGMENTS}

The TEXTOR interferometer/polarimeter was designed and built by $\mathrm{H}$. Soltwisch, who made the first current density measurements and coordinated the sawtooth research on TEXTOR until 1995. We acknowledge valuable contributions of A. Krämer-Flecken to the investigation of the influence of plasma rotation on the sawtooth period.

\section{REFERENCES}

1. A. J. H. DONNÉ et al., "Overview of Core Diagnostics for TEXTOR,” Fusion Sci. Technol., 47, 220 (2005).

2. H. R. KOSLOWSKI and H. SOLTWISCH, Fusion Eng. Des., 34-35, 143 (1997).

3. H. SOLTWISCH and EQUIPE TFR, Infrared. Phys., 21, 287 (1981).
4. S. L. VON GOELER, W. STODIEK, and N. SAUTHOFF, Phys. Rev. Lett., 33, 1201 (1974).

5. B. B. KADOMTSEV, Fiz. Plazmy, 1, 710 (1975); Sov. J. Plasma Phys., 1, 389 (1976).

6. J. O'ROURKE, Plasma Phys. Control. Fusion, 33, 289 (1991).

7. R. C. WOLF, J. O'ROURKE, A. W. EDWARDS, and M. VON HELLERMANN, Nucl. Fusion, 33, 663 (1993).

8. B. W. RICE, Rev. Sci. Instrum., 63, 5002 (1992).

9. B. W. RICE and E. B. HOOPER, Nucl. Fusion, 34, 1 (1994).

10. F. M. LEVINTON, Rev. Sci. Instrum., 63, 5157 (1992).

11. L. ZENG, D. L. BROWER, and Y. JIANG, Plasma Phys. Control. Fusion, 39, 591 (1996).

12. D. WRÓBLEWSKI and L. L. LAO, Phys. Fluids B, 3, 2877 (1991).

13. D. WRÓBLEWSKI and L. L. LAO, Rev. Sci. Instrum., 63, 5140 (1992).

14. J. A. WESSON, Plasma Phys. Control. Fusion, 28, 243 (1986).

15. J. RAPP et al., Plasma Phys. Control. Fusion, 39, 1615 (1997).

16. H. R. KOSLOWSKI, G. FUCHS, A. KRÄMER-FLECKEN, J. RAPP, and TEXTOR TEAM, Plasma Phys. Control. Fusion, 39, B325 (1997).

17. J. ONGENA et al., Phys. Scr., 52, 449 (1995).

18. H. SOLTWISCH, "Current Density Profiles and Sawtooth Activity in TEXTOR," Contributions to High Temperature Plasma Physics, p. 471, K. H. SPATSCHEK and J. UHLENBUSCH, Eds., Akademie, Berlin, Germany (1994).

19. H. SOLTWISCH, Rev. Sci. Instrum., 57, 1939 (1986).

20. H. SOLTWISCH, W. STODIEK, J. MANICKAM, and J. SCHLÜTER, Proc. 11th Int. Conf. Plasma Phys. and Control. Nucl. Fusion Res., p. 263, International Atomic Energy Agency, Vienna, Austria (1987).

21. YU. U. ESIPTCHUK and K. A. RAZUMOVA, Plasma Phys. Control. Fusion, 28, 1253 (1986).

22. H. SOLTWISCH, Rev. Sci. Instrum., 59, 1599 (1988).

23. H. SOLTWISCH and H. R. KOSLOWSKI, Plasma Phys. Control. Fusion, 39, A341 (1997).

24. K. ITOH, S.-I. ITOH, H. SOLTWISCH, and H. R. KOSLOWSKI, Plasma Phys. Control. Fusion, 39, 1033 (1997).

25. A. J. LICHTENBERG, K. ITOH, S.-I. ITOH, and A. FUKUYAMA, Nucl. Fusion, 32, 495 (1992).

26. B. UNTERBERG et al., "The Radiative Improved Mode in TEXTOR: Power Exhaust and Improved Confinement at High Density," Fusion Sci. Technol., 47, 187 (2005)

27. W. KERNER, J. P. GOEDBLOED, G. T. A. HUYSMANS, S. POEDTS, and E. SCHWARZ, J. Comp. Phys., 142, 271 (1998).

28. S. GÜNTER, A. GUDE, H. R. KOSLOWSKI, M. MARASCHEK, S. D. PINCHES, S. SESNIC, Q. YU, and ASDEX UPGRADE TEAM, Nucl. Fusion, 39, 1793 (1999).

29. H. R. KOSLOWSKI, H. SOLTWISCH, and W. STODIEK, Plasma Phys. Control. Fusion, 38, 271 (1996).

30. M. F. F. NAVE et al., Proc. 31st EPS Conf. Plasma Physics, London, United Kingdom, June 28-July 2, 2004, P1-162, European Physical Society (2004). 